\title{
The use of live virtual guides in educational applications
}

\author{
Vedad Hulusic \\ Sarajevo School of Science and Technology \\ Sarajevo \\ Bosnia and Herzegovina \\ Email:vedad.hulusic@ssst.edu.ba
}

\author{
Selma Rizvic \\ Sarajevo School of Science and Technology \\ Sarajevo \\ Bosnia and Herzegovina \\ Email: selma.rizvic@ssst.edu.ba
}

\begin{abstract}
Serious games are becoming increasingly popular in education, science, medicine, religion, engineering, and some other fields. Additionally, serious heritage games, including virtual reconstructions and museums, provide a good environment for a synthesis of serious games and cultural heritage. This may be used for education in the form of edutainment, comprising various techniques, such as storytelling, visual expression of information, interactivity and entertainment [1]. This paper demonstrates a new concept of using live virtual guides in a Flash environment for cultural heritage virtual reconstruction. A pilot user study compares another approach using the X3D environment, highlighting the advantages and disadvantages of our concept. The introduced results can be easily adopted for serious games development.
\end{abstract}

Keywords-virtual guides; cultural heritage; virtual reconstruction;

\section{INTRODUCTION}

For many years, games were considered only as an entertainment medium. However, serious gaming introduced several new aspects of gaming, including education, health training, scientific simulations, historical presentations, etc. Serious games, such as Microsoft Flight Simulator or Global Conflict: Palestine, can offer the same type of gameplay as commercial off-the-shelf games, or they can have different type of interaction and presentation, such as Houthoff $\mathrm{Bu}$ ruma - The Game.

One subcategory of serious games are serious heritage games [1]. These games involve cultural heritage objects or sites, usually in a form of virtual reconstructions or virtual museums. The former is typically used either for rebuilding non-existing objects or sites, or for digital $2 \mathrm{D}$ or $3 \mathrm{D}$ representation of an important existing object or site. The latter is used for popularisation of real museums providing world wide availability via internet or for allowing better examination of artefacts that are for example prohibited from touching. Additionally, in this type of environments, avatars or virtual guides may be employed for storytelling, which enhances the user experience by providing a higher level of immersion and presenting the important information in a narrative form.

In this paper we present a novel way of combining live virtual guides with computer generated heritage sites within Flash environment. The application is optimised for web presentations and does not require any additional software installations. Furthermore, this concept allows offline rendering. Since, even on the high-end machines it is still not possible to perform physically-based rendering at satisfactory interactive frame rates, our concept enables us to prerender images using physically-based rendering at no cost for the user.

The paper is organised as follows: in Section II we give an overview of the work done so far on virtual reconstructions, virtual museums, digital storytelling and virtual guides; Section III briefly describes the project of the virtual reconstruction of the Church of the Holy Trinity in Mostar; in Section IV our concept is described, through the steps of the virtual environment creation; in Section $\mathrm{V}$ the pilot user study is presented, together with the results; finally, the conclusions and the future work are given in section VI.

\section{RELATED WORK}

Virtual Reality(VR) environments, as we know it today, have been used for almost half a century, starting with the Morton Heilig's Sensorama in 1962, which is considered to be the first immersive, multi-sensory device. With the advancement of the technology and computing algorithms, the VR field progressed, producing more realistic and interactive environments and applications, such as [2], [3], [4]. However, these advances may not be satisfactory for conveying information to the user. This means that, although the virtual environment may be highly realistic and immersive, important information might not reach users in an understandable and desired way. An efficient way of teaching, instructing or informing users about some objects, events, facts or people is using digital storytelling [5], [6]. This technique helps involve the audience as active participants and drives the development of a plot.

\section{A. Virtual reconstruction of cultural heritage}

Virtual reconstruction of cultural heritage sites is extremely important for preservation and presentation of both non-existing and existing historical sites and stories, culture, architecture, customs and moral values. There are numerous examples of virtual reconstructions of different historical sites from all around the world [7], [8], [9], [10], [11], [12], 
[13], [14]. Another type of promotion and preservation of cultural heritage is the creation of virtual museums [15], [16], [17], [18], [19], [20], [21], [22], [23], [24], [25], [26], [27], [28], [29]. Virtual museums allow remote visitors to experience and explore the museums using web presentations, attract potential visitors to visit the real museums and inform the visitors in the actual museums what they can see in the museum. It also provides a detailed exploration of some preserved artefacts which had to be conserved.

\section{B. Virtual guides}

There are multiple forms of conveying information to a user, such as text, audio, video, animated 3D characters, real or virtual avatars, etc. The results from a user study by Sadzak et al. showed that live virtual guides are more effective and preferable for users than computer generated virtual guides. [30]. Furthermore, forms of storytelling have been proven as a good concept for information conveyance in virtual environments [31], [32]. Therefore, in this paper we used digital storytelling performed by a live virtual guide, in order to tell a user the stories about the reconstructed object and its important elements.

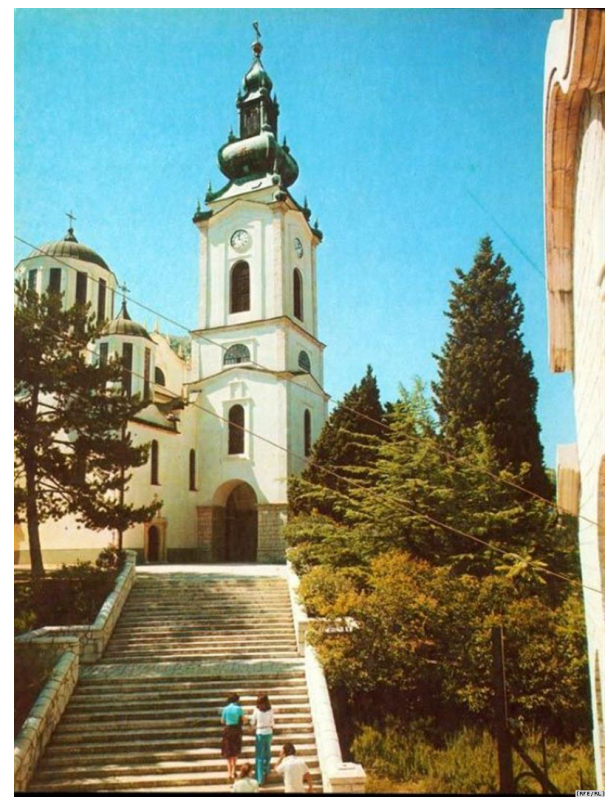

Figure 1. The church as it looked before its destruction.

\section{ViRTUAL RECONSTRUCTION OF THE CHURCH OF THE HOLY TRINITY IN MOSTAR}

The Church of the Holy Trinity in Mostar (Figure 1) was one of the most important and most significant religious and cultural objects in Bosnia and Herzegovina and the Balkans. This Orthodox church was completely destroyed in 1992, see Figure 2. In order to promote its beauty and significance, as well as to encourage its physical reconstruction, a virtual reconstruction has been done [33], see Figure 3. The project of the virtual reconstruction included: creating a 3D model of the church interior and exterior; capturing the High Dynamic Range (HDR) panoramic photograph of the church environment; developing a web and offline presentation with historical information, photo gallery, video of the church devastation, X3D interactive virtual model and a digital story with the live virtual guides; and printing a 3D prototype of the model [14]. The project aroused significant media attention and the Orthodox religious community was very pleased with the results.

However, there were a couple of drawbacks in the project. The first one was its data size. This is particularly considerable in countries with lower internet speeds, such as Bosnia and Herzegovina. Table I shows the model size and the download times, both for the outdoor and indoor part of the model. Although the 3D model was partially optimised, the geometry could be further optimised using polygon reduction methods. However, this process usually requires significant amount of time, as it can not be fully automated. Furthermore, the geometry itself might not be suitable for optimisation (holes in the objects, non-manifold geometry, duplicated and overlapping vetrices, edges or faces, etc.), which, therefore, requires geometry clean-up and reconstruction.

The second drawback is the software requirement. Namely, a user has to install X3D player or browser plug-in for viewing the model. This is not very popular amongst internet users, as it consumes time, requires certain level of computer literacy and represents a security risk, as the user can not be sure if the program could harm his or her machine.

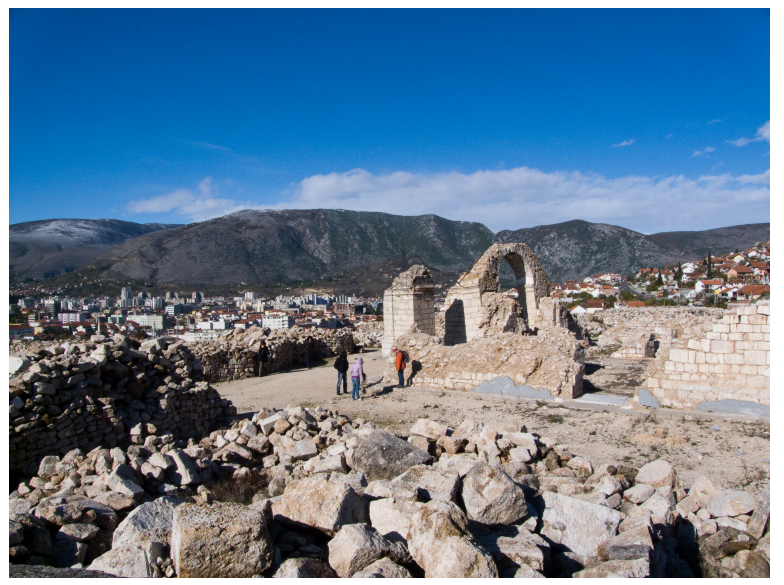

Figure 2. The church was completely destroyed in 1992. The figure shows the current state of the object.

\section{CReating the virtual enVironment in Flash}

In order to improve the performance of the virtual environment, another approach was employed. Instead of exporting 


\begin{tabular}{|c||c|c|c|c|c|}
\hline \multicolumn{1}{|c||}{} & \multicolumn{2}{c|}{ Outdoor } & \multicolumn{2}{c|}{ Indoor } & Outdoor + Indoor \\
\hline \multicolumn{1}{|c||}{ X3D } & Total size & Initial size & Total size & Initial size & Total \\
\hline (download time) & $58 \mathrm{MB}$ & $32 \mathrm{MB}$ & $45 \mathrm{MB}$ & $28.8 \mathrm{MB}$ & $103 \mathrm{MB}$ \\
\hline Flash & $(155 \mathrm{~s})$ & $(85 \mathrm{~s})$ & $(120 \mathrm{~s})$ & $(77 \mathrm{~s})$ & $(275 \mathrm{~s})$ \\
$($ download time $)$ & $5.6 \mathrm{MB}$ & $5.6 \mathrm{MB}$ & $5.1 \mathrm{MB}$ & $5.1 \mathrm{MB}$ & $10.7 \mathrm{MB}$ \\
& $(15 \mathrm{~s})$ & $(15 s)$ & $(14 s)$ & $(14 s)$ & $(29 s)$ \\
\hline
\end{tabular}

Table I

THE COMPARISON OF THE DATA SIZE AND DOWNLOAD TIME FOR THE X3D AND OUR CONCEPT (FLASH). THE DOWNLOAD SPEED USED FOR THE MEASUREMENT WAS 3 MBPS.

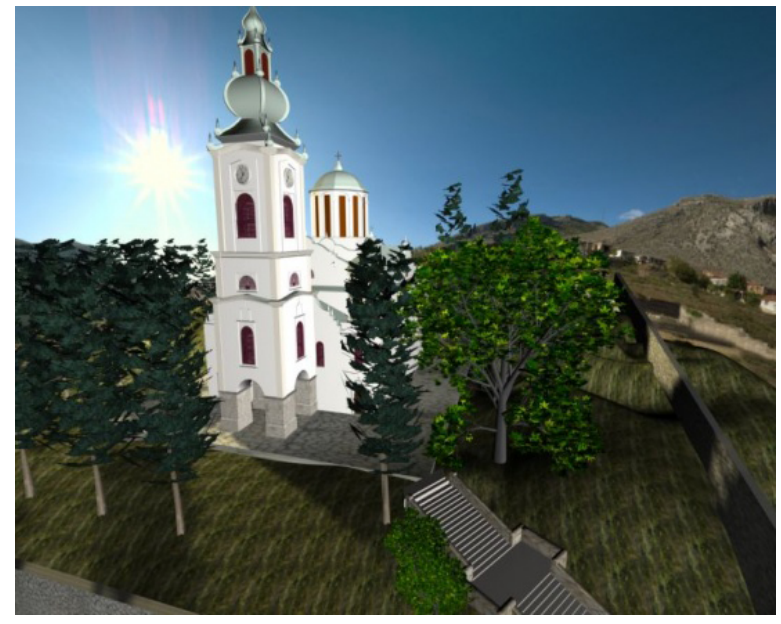

Figure 3. A rendered image of the virtually reconstructed church.

the 3D model to X3D, the panoramic images were rendered directly from Autodesk Maya, using the MentalRay renderer. Since Maya does not provide a panoramic lens by default, the latlong lens MentalRay lens shader was downloaded and used [34]. The shader enables a full 360/180 rendering of a scene in latitude/longitude format. We decided to render the indoor and outdoor scenes at three view points each, see Figure 4. During the scene set up, it was possible to further improve the appearance of the scene by adjusting the geometry, lights and materials. However, we tried to make as little intervention on the $3 \mathrm{D}$ model as possible.
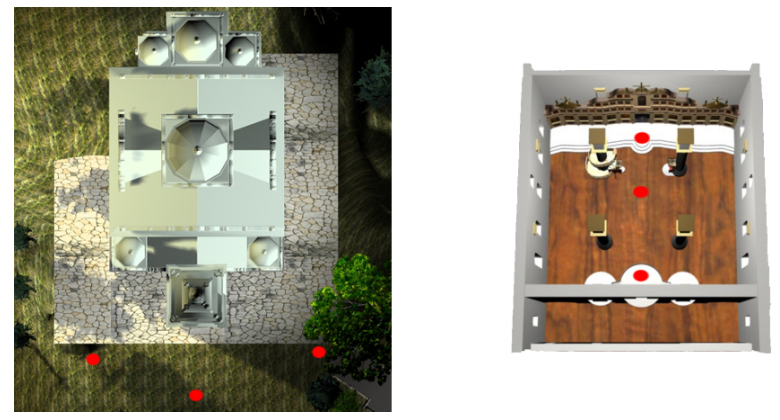

Figure 4. The rendering view points outdoors(left) and indoors(right) marked with the red dots.
The virtual guide was recorded against a green screen background and keyed out with the alpha channel using the Keylight plug-in in Adobe After Effects CS5, see Figure 5. The output was saved as a lossless Quicktime movie and then recoded into Flash (.swf) format using Adobe Media Encoder CS5.

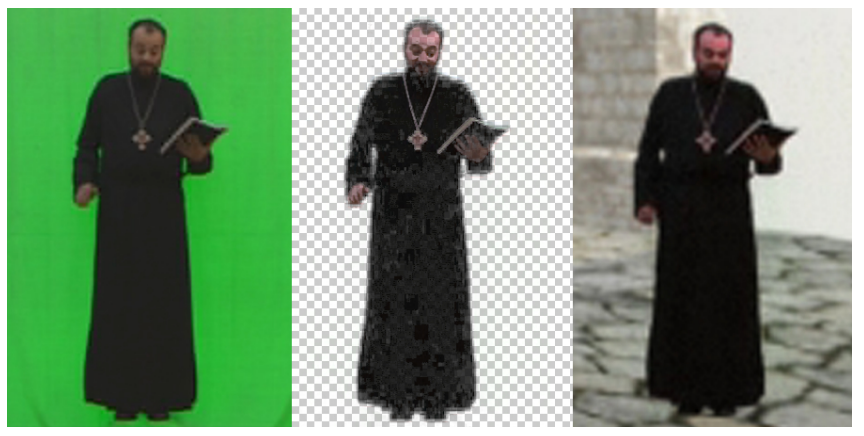

Figure 5. Keying the footage: virtual guide against the green screen (left); virtual guide keyed out with alpha channel (middle); virtual guide superimposed on the virtual model (right).

For the virtual environment and user interface Tourweaver 5.00 Professional Edition was used. This software allowed us to merge the prepared images, create navigation, embed virtual guides and export it for both stand-alone (executable) and web presentation (Flash). We started with a blank new project, and imported the images as scenes. Then, on corresponding scenes we added Flash Media and chose the virtual guide, see Figure 6.

In the next step we added the Map Viewer and assigned it the image of the map. On each map the Radar Hotspots were set up, see Figure 4. For easier navigation, i.e. moving across the view points, Thumbnails were added at the bottom of the screen. There were three thumbnails, one for each view point, on both interior and exterior model. The final application looked as in Figures 7 and 8.

The last step in the process was to export i.e. publish the environment. Publishing options offer Flash VR and Flash VR(exe) type of exportation. The data size and the corresponding download times of the indoor and outdoor models are given in Table I. 


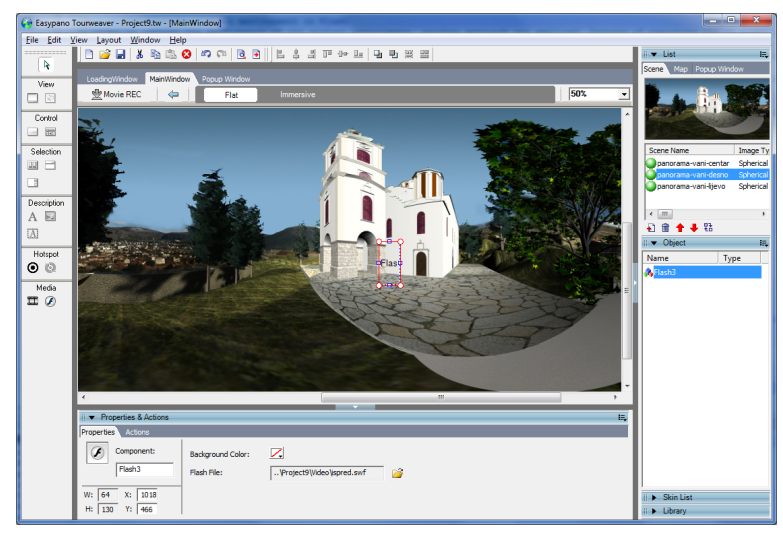

Figure 6. The proces of creating the virtual environment.

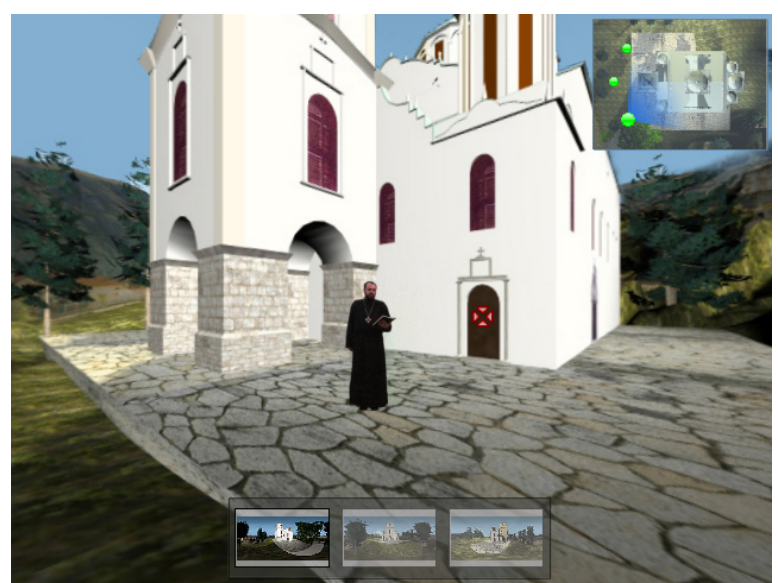

Figure 7. The exterior of the virtual environment.

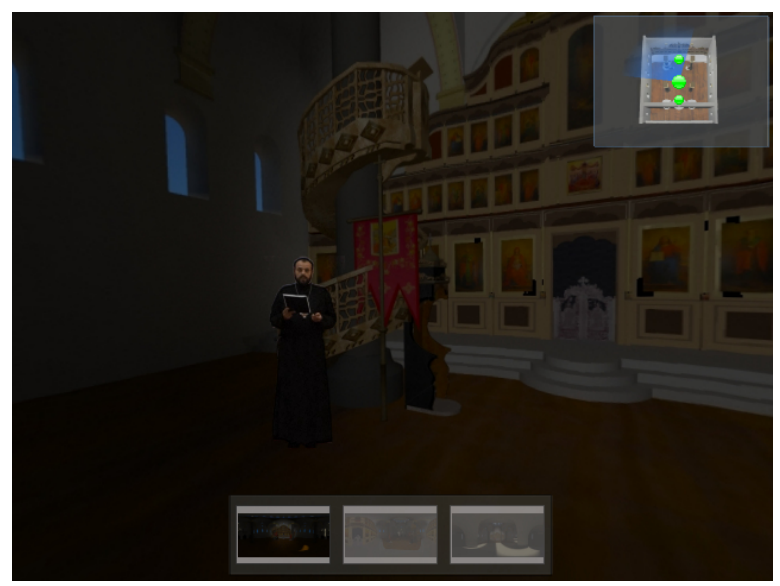

Figure 8. The interior of the virtual environment.

\section{USER STUDY: X3D VS. FLASH}

As shown in Section III, using our method the data size was decreased around ten times, which speeds up the loading process dramatically. This factor might be significant, particularly for users with lower internet speeds. In order to compare the two environments - the X3D and our concept, we made a pilot user study with 10 participants. There were 5 male and 5 female participants, aged from 24 to 43, with an average of 30 . All of them had normal or corrected to normal vision and no hearing impairments. The participants were sent the instructions and the questionnaire by email. They were asked to send the questionnaire back upon completion. All participants reported to have broadband connection with varying download and upload speeds. Two participants, one male and one female, reported that they could not run the X3D environment. This might be due to low download speed (one of the two participants reported bandwidth of 512/256 bps) or some other technical issues such as firewall. These two participants were excluded from the results evaluation.

The results gained from the pilot study are presented in Table II. From the results we can see that the users were more satisfied with the Flash implementation, comparing to X3D. They reported higher loading speed, better navigation, graphics and virtual guides in the Flash application. Additionally, $50 \%$ of the participants stated that they do not mind installing new software and $50 \%$ prefer not to install any new software. Nevertheless, some users expressed concerns regarding the freedom of navigation, stating that the $\mathrm{x} 3 \mathrm{D}$ environment offers better browsing possibilities.

Although, the pilot study was conducted with a small sample size, and therefore does not provide a statistically significant evidence, the results represent a good indication of the potential of using live virtual guides in Flash environment for cultural heritage virtual reconstruction.

\section{CONCLUSIONS AND FUTURE WORK}

The technologies commonly associated with computer games are not any more solely an entertainment medium. Instead, they could be a significant factor in education, medical and scientific applications and simulations, and historical presentations. In this paper we explored how live virtual guides could be used for storytelling purposes in a virtual cultural heritage reconstruction scenario. We combined the live virtual guide with the alpha channel onto panoramic images in Flash environment. The model was then compared with the same scenario implemented with the X3D virtual environment. The Flash implementation introduced many benefits comparing to the X3D application. The main advantages were data size, download speed, offline rendering capability, better image quality, and easier navigation in the environment. The main drawback of our approach is the navigational restriction to predefined view points in the environment. This, however, might be considered as a minor issue comparing to the achieved enhancements. 


\begin{tabular}{|l|c|c|}
\hline Question & X3D & Flash \\
\hline Which application loads faster? & 0 & 8 \\
\hline Which application is easier to navigate through (explore the environment)? & 1 & 7 \\
\hline Which application has better graphics (looks better)? & 1 & 7 \\
\hline What virtual guide (avatar) did you prefer? & 1 & 7 \\
\hline Which application do you prefer in overall? & 0 & 8 \\
\hline \hline How did you feel about installing new software onto your machine: & \\
- I like installing new software & 0 \\
- I do not mind installing new software & 4 \\
- I prefer not to install new software & 4 \\
- I never install any new software & 0 \\
\hline
\end{tabular}

Table II

THE RESULTS FROM THE PILOT USER STUDY, SHOWING THE NUMBER OF GIVEN ANSWERS.

The results from the user study imply that the loading speed, dependent mostly on the data size, and the possibility to run the environment is more important than the ability to freely walk around the object. Furthermore, with the Flash environment, it is possible to add as many view points as we like, and to link them to important objects or spots. That would give more freedom and spatial width to a user while browsing the environment. Additionally, for the comparison purpose, the 3D model for the Flash implementation was rendered using almost the same scene setup as for the X3D application, in order to achieve similar appearance of the outcome. Nevertheless, the models could be rendered with higher visual quality, adjusting the geometry, lighting, material and rendering settings within Maya or similar modeling and/or rendering software. Those steps could have been directly implemented in the model creation pipeline, if the model was intended for high-fidelity rendering purposes, which, in that case, would not require any additional effort.

This is, to the best of our knowledge, the first attempt to use live virtual guides in a Flash environment for cultural heritage virtual reconstruction. Therefore, it represents a further step from the traditional non-interactive narrative content in computer games: the narrator is a live person and the environment is interactive - one can explore it while listening to the narrator. This concept could be used for creating educational serious games, such as quizzes or puzzle games, where storytelling is important aspect of the game. Additionally, it could be exceptionally suitable for virtual reconstructions and virtual museum applications.

In the future a user study with more participants should be undertaken, comparing different concepts in terms of storytelling, interactivity, information conveyance, audio-visual quality and gameplay. Furthermore, it would be valuable to make a serious game using our concept and evaluate the same elements through the actual gameplay.

\section{ACKNOWLEDGMENT}

The authors would like to thank all the volunteers who participated in the study.

\section{REFERENCES}

[1] E. F. Anderson, L. McLoughlin, F. Liarokapis, C. Peters, P. Petridis, and S. de Freitas, "Serious games in cultural heritage," in VAST 2009: 10th International Symposium on Virtual Reality, Archaeology and Cultural Heritage - VASTSTAR, Short and Project Proceedings. Faculty of ICT, University of Malta, 2009, pp. 29-48, state-of-the-Art-Report.

[2] A. Gaitatzes, D. Christopoulos, and M. Roussou, "Reviving the past: cultural heritage meets virtual reality," in VAST '01: Proceedings of the 2001 conference on Virtual reality, archeology, and cultural heritage. New York, NY, USA: ACM, 2001, pp. 103-110.

[3] A. Johnson, J. Leigh, B. Carter, J. Sosnoski, and S. Jones, "Virtual harlem," IEEE Computer Graphics and Applications, vol. 22, pp. 61-67, 2002.

[4] S.-C. Yeh, B. Newman, M. Liewer, J. Pair, A. Treskunov, G. Reger, B. O. Rothbaum, J. Difede, J. Spitalnick, R. McLay, T. D. Parsons, and A. A. Rizzo, "A virtual iraq system for the treatment of combat-related posttraumatic stress disorder," in $V R, 2009$, pp. 163-170.

[5] S. Brown, I. Ladeira, C. Winterbottom, and E. Blake, "The effects of mediation in a storytelling virtual environment," in In Virtual Storytelling: Using Virtual Reality Technologies for Storytelling (2003), Springer-Verlag. Proc. 2nd International Conference ICVS, 2003, pp. 102-111.

[6] I. Ladeira and E. H. Blake, "Virtual san storytelling for children: Content vs. experience," in VAST, 2004, pp. 223231.

[7] A. Foni, G. Papagiannakis, and N. Magnenat-Thalmann, "Virtual hagia sophia: Restitution, visualization and virtual life simulation," in UNESCO World Heritage Congress Proceedings, October 2002.

[8] V. Sundstedt, A. Chalmers, and P. Martinez, "High fidelity reconstruction of the ancient egyptian temple of kalabsha," in AFRIGRAPH '04: Proceedings of the 3rd international conference on Computer graphics, virtual reality, visualisation and interaction in Africa. New York, NY, USA: ACM, 2004, pp. 107-113.

[9] P. Debevec, "Making "the parthenon"," 2004. 
[10] D. Gutierrez, F. J. Seron, J. A. Magallon, E. J. Sobreviela, and P. Latorre, "Archaeological and cultural heritage: bringing life to an unearthed muslim suburb in an immersive environment," Journal of Cultural Heritage, vol. 5, no. 1, pp. 63 - 74, 2004. [Online]. Available: http:// www.sciencedirect.com/science/article/B6W6G-4BY308Y-7/ 2/5914a86f62db9bfe79a5c2844600f6e5

[11] G. P. Earl, "Wandering the house of the birds: reconstruction and perception at roman italica," in VAST '05: Proceedings of the 2005 conference on Virtual reality, archeology, and cultural heritage, short papers, 2005.

[12] S. Rizvic, A. Sadzak, E. Buza, and A. Chalmers, "Virtual reconstruction and digitalization of cultural heritage sites in bosnia and herzegovina," in SEEDI 2007, Review of the National Center for Digitization. Faculty of Mathematics, Belgrade, Serbia, 2008, pp. 82-90.

[13] B. Frischer, D. Abernathy, G. Guidi, J. Myers, C. Thibodeau, A. Salvemini, P. Müller, P. Hofstee, and B. Minor, "Rome reborn," in SIGGRAPH '08: ACM SIGGRAPH 2008 new tech demos. New York, NY, USA: ACM, 2008, pp. 1-1.

[14] B. Ramic-Brkic, Z. Karkin, A. Sadzak, D. Selimovic, and S. Rizvic, "Augmented real-time virtual environment of the church of the holy trinity in mostar," in Proceedings of the 2009 ACM/Eurographics International Symposium on Virtual Reality, Archaeology and Cultural Heritage, 2009, pp. 141148.

[15] “The uffizi (florence)," http://musa.uffizi.firenze.it/. [Online]. Available: http://musa.uffizi.firenze.it/

[16] "The tower of pisa," http://www.compart-multimedia.com/. [Online]. Available: http://www.compart-multimedia.com/ virtuale/

[17] "The national gallery (london)," http://www.nationalgallery. org.uk. [Online]. Available: http://www.nationalgallery.org. uk/

[18] "The metropolitan museum (new york)," http: //www.metmuseum.org/. [Online]. Available: http://www. metmuseum.org/

[19] “Louvre," http://www.louvre.fr/.

[20] "Hermitage museum," http://www.hermitage.ru.

[21] "National museum of the american indian," http://www.nmai. si.edu/, 2002.

[22] “Arco," http://www.arco-web.org/, 2003. [Online]. Available: http://www.arco-web.org/

[23] "Europeana," http://group.europeana.eu/, 2008. [Online]. Available: http://group.europeana.eu/

[24] "Athena," http://www.athenaeurope.org/, 2008. [Online]. Available: http://www.athenaeurope.org/

[25] "Virtual museum of bosnian traditional objects," http://www. muzejsarajeva.ba/, 2009.
[26] "Digital catalogue of stecaks," http://h.etf.unsa.ba/ dig-katalog-stecaka, 2009.

[27] "Virtual national museum of bosnia and herzegovina," http: //projects.etf.unsa.ba/ vmuzej/.

[28] "Virtual museum of the sarajevo assassination," http://h.etf. unsa.ba/vmuzej-atentata.

[29] "The virtual museum of canada," http://www. museevirtuel-virtualmuseum.ca/. [Online]. Available: http: //www.museevirtuel-virtualmuseum.ca/

[30] A. Sadzak, S. Rizvic, C. Dalton, and A. Chalmers, "Information perception in virtual heritage storytelling using animated and real avatars," in Spring Conference on Computer Graphics, 2007.

[31] A. Sadzak, S. Rizvic, and A. Chalmers, "The influence of storytelling quality on the human perception of computer animation," in International Symposium on Virtual Reality, Archaeology and Cultural Heritage (VAST 2007), 2007.

[32] S. Rizvic and A. Sadzak, "Digital storytelling - representation of bosnian intangible heritage in the virtual sarajevo project," in International Symposium on Virtual Reality, Archaeology and Cultural Heritage (VAST 2008), Braga, Portugal, 2008.

[33] Z. Karkin and S. Rizvic, "Virtuelna 3d rekonstrukcija crkve svete trojice u mostaru," in International Symposium on Digitalization of Cultural Heritage in Bosnia and Herzegovina, 2008.

[34] R. Habel. Create your own hdr environment map in maya/mental ray for image based lighting. http://www. vi-motion.de/latlong \_Tutorial/. 